\title{
Diseño y simulación de un molino compacto para polietileno tereftalato
}

\section{Design and simulation of a compact polyethylene terephthalate mil}

DOI: $10.46932 / \mathrm{sfjdv} 3 \mathrm{n} 1-018$

Received in: Dec 30st, 2021

Accepted in: Jan 1th, 2022

\author{
Miguel Ángel Madrigal Cardiel \\ Dr. En Ciencias de la Educación. \\ Tecnológico Nacional de México.
}

Instituto Tecnológico Superior P'urhépecha, División de Ingeniería Industrial, Carretera Carapan-

Uruapan Km. 31.5, Cherán, Michoacán. C.P. 60270, Tel. (443) 2921023 y (443) 2921026

E-mail: miguel.mc@purhepecha.tecnm.mx

\section{Silvano Velázquez Roque}

Maestro en Ciencias en la Ingeniería Física.

Tecnológico Nacional de México.

Instituto Tecnológico Superior P'urhépecha, División de Ingeniería Industrial, Carretera Carapan-

Uruapan Km. 31.5, Cherán, Michoacán. C.P. 60270, Tel. (443) 2921023 y (443) 2921026

E-mail: silvano.rv@purhepecha.tecnm.mx

\section{Jesús Maldonado Paleo}

Maestro en Ingeniería Eléctrica.

Tecnológico Nacional de México.

Instituto Tecnológico Superior P'urhépecha, División de Ingeniería Biomédica, Carretera Carapan-

Uruapan Km. 31.5, Cherán, Michoacán. C.P. 60270, Tel. (443) 2921023 y (443) 2921026

E-mail: jesus.mp@purhepecha.tecnm.mx

\section{Ulises Zurita Luna}

Maestro en Ciencias en Ingeniería química.

Tecnológico Nacional de México.

Instituto Tecnológico Superior P'urhépecha, División en Energías Renovables. Carretera CarapanUruapan

Km. 31.5, Cherán Michoacán. C.P. 60270

E-mail: ulises.zl@purhepecha.tecnm.mx

\section{Juan Damián Hernández}

Ingeniero Industrial.

Tecnológico Nacional de México.

Instituto Tecnológico Superior P'urhépecha, División de Ingeniería Industrial, Carretera Carapan-

Uruapan Km. 31.5, Cherán, Michoacán. C.P. 60270, Tel. (443) 2921023 y (443) 2921026

E-mail: juan.dh@purhepecha.tecnm.mx

\section{RESUMEN}

En este trabajo, se presenta el diseño de un molino compacto para triturar envases de polietileno tereftalato (PET), con el objetivo de que en una siguiente etapa se pueda implementar de manera física y hacer frente a la problemática que se tiene por la generación y uso excesivo de este tipo de envases, que si bien es 
cierto ofrece una gran ventaja para el envasado de bebidas, cuando no se les da un buen tratamiento se convierten en residuos que constituyen focos importantes de contaminación. Dentro del Instituto Tecnológico Superior P'urhépecha (ITSP), se observa la presencia de estos residuos en un promedio de $5 \mathrm{Kg}$ diarios, a pesar de la implementación de la normativa NOM-ISO14000, por tal motivo se pensó en una solución para dar un tratamiento integral a estos residuos, siendo la primera etapa el diseño del molino, debido a que en la actualidad solo existen molinos o trituradoras de gran escala cuya adquisición es costosa. Por otro lado, en el mercado existen gran variedad de paquetes computacionales para el dibujo de piezas mecánicas, como por ejemplo el Autocad, sin embargo se optó por el uso del software SolidWorks, porque además de que permitió el dibujo de las piezas mecánicas de una manera relativamente sencilla, también brindó la opción de realizar un ensamble entre ellas, así como realización de cálculos de elemento finito, observando de esta manera las condiciones de operación del molino, permitiendo así contar con una validación del diseño, lo cual servirá para su implementación física. Los materiales utilizados en el diseño, son de uso convencional y de fácil adquisición y por su tamaño puede resultar económicamente accesible para su implementación dentro del ITSP o bien en alguna otra institución educativa pública o privada.

Palabras Clave: Solidworks, Molino, Pet, Diseño, Simulación.

\begin{abstract}
In this work, the design of a compact mill to crush polyethylene terephthalate (PET) containers is presented, with the objective that in a next stage it can be implemented physically and face the problems that exist due to the generation and excessive use of this type of packaging, which, although it is true, offers a great advantage for the packaging of beverages, when they are not properly treated, they become waste that constitutes major sources of contamination. Within of Instituto Tecnológico Superior P'urhépecha (ITSP), the presence of these residues is observed in an average of $5 \mathrm{Kg}$ per day, despite the implementation of the NOM-ISO14000 regulation, for this reason a solution was thought to give a treatment integral to these residues, the first stage being the design of the mill, since at present there are only large-scale mills or crushers whose acquisition is expensive. On the other hand, in the market there are a great variety of computer packages for the drawing of mechanical parts, such as Autocad, however, the use of SolidWorks software was chosen, because in addition to allowing the drawing of mechanical parts relatively simple way, it also offered the option of making an assembly between them, as well as carrying out finite element calculations, thus observing the operating conditions of the mill, thus allowing a design validation, which will serve for its implementation physical. The materials used in the design are of conventional use and easy to acquire, and due to their size, they can be economically accessible for implementation within the ITSP or in some other public or private educational institution.
\end{abstract}

Keywords: SolidWorks, Mill, Pet, Design, Simulation.

\title{
1 INTRODUCCIÓN
}

La presente investigación se refiere al diseño de un molino compacto para triturar envases de polietileno tereftalato (PET). Entendiendo el diseño en ingeniería como “el proceso de aplicación de varias técnicas y principios científicos, con la finalidad de definir un dispositivo, un proceso o un sistema, con el detalle suficiente que permita su realización"[1]. La simulación en el software SolidWorks permite "someter fácilmente sus diseños a las mismas condiciones a las que se expondrán en el mundo real, permitiendo mejorar la calidad de sus productos, optimizar las geometrías y materiales, y además reducir 
costos tanto en tiempo como en el ahorro de prototipo para pruebas reales"[2]. El tereftalato de polietileno (PET) es un tipo de plástico muy usado en envases de bebidas y textiles [3]. Estos envases de PET son los que se presentan en la mayoría de las bebidas azucaradas que tienen una gran demanda en el mercado. El problema del plástico PET es que su producción es muy alta, y su reciclaje o reutilización se considera demasiada baja.

En la actualidad sólo existen molinos industriales de gran capacidad y en consecuencia son de gran tamaño y costosos. Se pretende que el molino sea de bajo costo, comparado con los molinos comerciales, además de que los materiales utilizados serán accesibles y fácil de conseguir en el mercado, así como las partes de la máquina tienen una forma geométrica sencilla y fácil de construir.

El objetivo principal de este trabajo es la simulación del diseño mecánico de un molino de bajo costo para triturar envases o botellas de PET, provenientes del Instituto Tecnológico Superior P'urhépecha (ITSP). Debido a que, en la institución, se genera gran cantidad de botes de plástico que provienen de productos alimenticios (agua, jugos, refrescos, etc.) una vez que se han utilizado, los depositan en los botes de separación para su posterior venta o en su defecto tirarlos hacia a la basura. Al igual que en el instituto existen otras instituciones con el mismo problema anteriormente mencionado, el desperdicio del PET. Para lograr el objetivo se recurren a las siguientes etapas del diseño; caracterización de las propiedades mecánicas del PET; propuestas de diseños; realizar el diseño más óptimo y crear la simulación en el software SolidWorks. Como el proyecto es ambicioso quedaría pendiente la fabricación de la máquina y la evaluación de ésta en otro momento.

\section{MATERIALES Y MÉTODOS}

La metodología que se empleó para esta investigación fue el proceso de diseño de Robert L. Norton [1]. Este proceso consta de diez pasos que se muestran en la tabla 1. En este trabajo solo se desarrolló hasta el proceso 8 . El proceso 9 y 10 se realizará en otra etapa de la investigación, que no está contemplada en el presente trabajo.

Tabla 1. El proceso de diseño [1].

\begin{aligned} & \hline Tabla 1 El proceso de diseño \\ & \hline 1 Identificación de la necesidad \\ & 2 Investigación preliminar \\ & 3 Planteamiento de la meta \\ & 4 Especificaciones de funcionamiento \\ & 5 Síntesis \\ & 6 Análisis \\ & 7 Selección de la solución \\ & 8 Diseño detallado \\ & 9 Prototipos y pruebas \\ & 10 Producción \\ & \hline\end{aligned}


El paso inicial, identificación de la necesidad, normalmente consiste en un planteamiento del problema vago y escueto. Para este caso fue el no aprovechamiento de los envases de PET, provenientes de la institución.

En el paso dos, investigación preliminar, se definió y se entendió por completo el problema a resolver, así como también se investigó de los molinos actuales en el mercado, y se llegó a la conclusión de que solo existen de gran tamaño y en consecuencia son costosos.

En el paso tres, planteamiento de la meta, se definió que la máquina debía de ser de medidas compactas, que los materiales se pudieran conseguir fácilmente en el mercado y como consecuencia se obtiene un molino cuyas características no se encuentran en los molinos del mercado actual.

En el paso cuatro, especificaciones del funcionamiento, se define lo que el sistema debe hacer. Para esta investigación fue que la máquina entregue como producto final escamas de PET, para su posterior utilización.

En el paso cinco, síntesis, es dónde se encontraron los enfoques de diseño alternativos que fueron posibles. Es donde se generó el mayor número de soluciones creativas. También se denomina el paso de ideación e invención —aquí se generó el mayor número viable de soluciones creativas.

En el paso seis, análisis, se aplicaron técnicas de análisis por medio del elemento finito en el software SolidWorks, principalmente la Teoría de Von Mises-Hencky o de energía de distorsión, que es una magnitud física proporcional a la energía de distorsión. En ingeniería estructural se usa en el contexto de las teorías de fallo como indicador de un buen diseño para materiales dúctiles.

La tensión de Von Mises puede calcularse a partir de las tensiones principales del tensor tensión en un punto de un sólido deformable, mediante la expresión:

$$
U=\frac{1}{2 E}\left[\sigma_{1}^{2}+\sigma_{2}^{2}+\sigma_{3}^{2}-2 v\left(\sigma_{1} \sigma_{2}+\sigma_{2} \sigma_{3}+\sigma_{1} \sigma_{3}\right)\right]
$$

Donde:

$U$, es la densidad de energía de deformación.

$E$, es el módulo de Young o elasticidad.

$\sigma_{1}, \sigma_{2}, \sigma_{3}$, son los esfuerzos principales.

$v$, es la razón de Poisson.

El esfuerzo efectivo de Von Mises $\sigma$ ’ se define como el esfuerzo de tensión uniaxial que crearía la misma energía de distorsión que la combinación real de los esfuerzos aplicados [1].

$$
\sigma^{\prime}=\sqrt{\sigma_{1}^{2}+\sigma_{2}^{2}+\sigma_{3}^{2}-\sigma_{1} \sigma_{2}-\sigma_{2} \sigma_{3}-\sigma_{1} \sigma_{3}}
$$

La ecuación (2) también, se puede escribir como. 


$$
S_{y}=\sqrt{\sigma_{1}^{2}+\sigma_{2}^{2}+\sigma_{3}^{2}-\sigma_{1} \sigma_{2}-\sigma_{2} \sigma_{3}-\sigma_{1} \sigma_{3}}
$$

Donde:

$S_{y}$, es el esfuerzo de fluencia del material.

Para el esfuerzo bidimensional, $\sigma_{2}=0$ y la ecuación (3) se reduce a

$$
S_{y}=\sqrt{\sigma_{1}^{2}-\sigma_{1} \sigma_{3}+\sigma_{3}^{2}}
$$

La ecuación bidimensional de la energía de distorsión (4) describe una elipse, la cual, al graficarse sobre los ejes $\sigma_{1}, \sigma_{3}$, queda como la de la figura 1. El interior de esta elipse define la región segura contra la fluencia bajo carga estática, para los esfuerzos biaxiales combinados [1]. Bajo esta teoria es que se analizaron las cuchillas o cortadores del molino, para no sufrir roptura.

Figura 1. Elipse normalizada de la energía de distorsión bidimensional, para la resistencia a la fluencia del material [1].

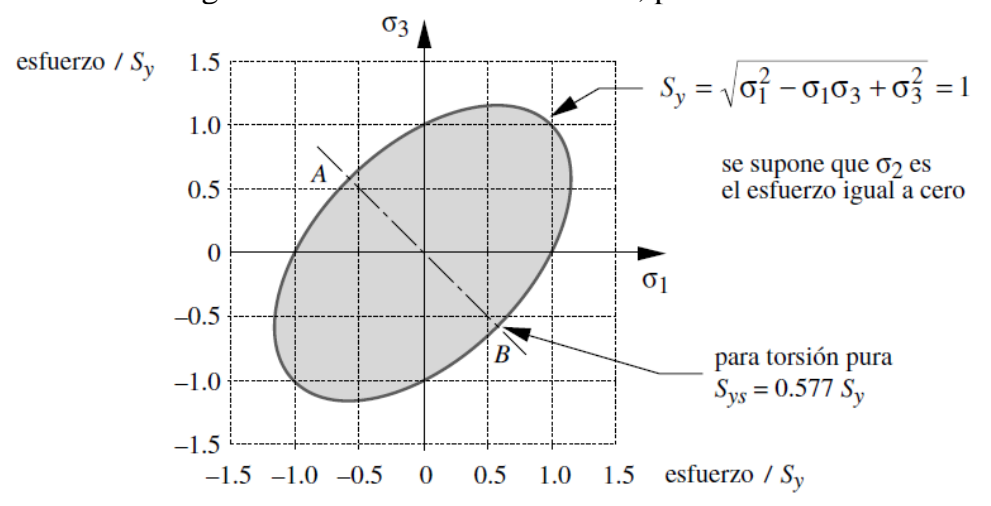

En el paso siete, selección de la solución, cuando el análisis técnico indica que se tienen algunos diseños potencialmente factibles, se debe seleccionar el mejor disponible para un diseño detallado, creación de prototipo y pruebas. El proceso de selección casi siempre implica un análisis comparativo de las soluciones de diseño disponibles [4]. Se realizó la comparación de diferentes diseños de cuchillas y se seleccionaron las más optimas en cuanto a diseño y su resistencia a la falla.

En el paso ocho, diseño detallado. Este paso en general incluye la creación de un conjunto completo de dibujos de ensamble detallados, o archivos de diseño asistido por computadora (CAD), por cada pieza utilizada en el diseño. Cada dibujo detallado debe especificar todas las dimensiones y las especificaciones de material necesarias para fabricar la pieza. Con estos dibujos (o archivos CAD) se necesita construir un modelo (o modelos) de prototipos para experimentos físicos [4]. Aquí se definieron las especificaciones técnicas del molino compacto, por ejemplo, la potencia del motor eléctrico, la transmisión de potencia por medio de poleas, diseño de poleas, tipo de bandas, el diseño de las cuchillas en el interior del molino, el tipo de material de nuestras cuchillas, y el tipo de base para sentar el molino. 


\section{DISCUSIÓN DE RESULTADOS}

\subsection{PARTES DE LA MÁQUINA DEL MOLINO.}

A continuación, se muestran las principales partes de la máquina del molino para triturar PET. La figura 2a, representa la vista frontal del juego de cuchillas, estas forman una figura tipo rehilete, el diámetro exterior es de $123 \mathrm{~mm}$. El cuadro en el interior es de lado de $25 \mathrm{~mm}$. El ángulo de la cuchilla forma $45^{\circ}$, debido a que, a ese valor, es cuando tenemos el cortante máximo. El espesor de las cuchillas es de $6.4 \mathrm{~mm}$. Existe un separador entre cada cuchilla que tiene un diámetro de $50 \mathrm{~mm}$ y en su interior tiene también un cuadrado, que mide cada lado de $25 \mathrm{~mm}$. En la figura $2 \mathrm{~b}$, se muestra la vista superior donde van montadas las cuchillas y los separadores, en total tenemos 32 cuchillas y 33 separadores, como consecuencia tiene una longitud de $363.20 \mathrm{~mm}$. Por último, la figura 2c, representa la vista isométrica del juego de cuchillas principales.

Figura 2. Juego de cuchillas principales.

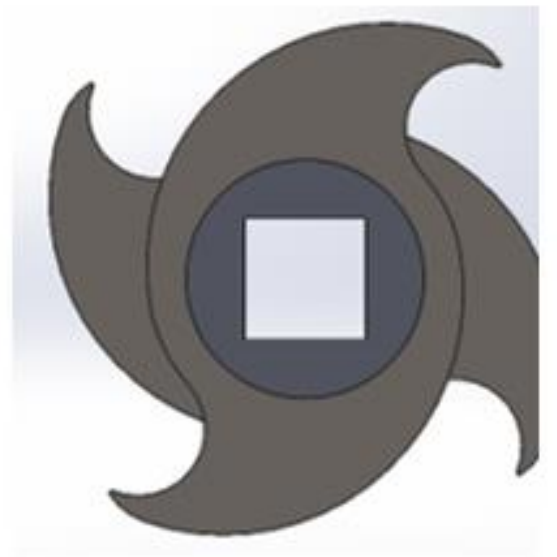

a)

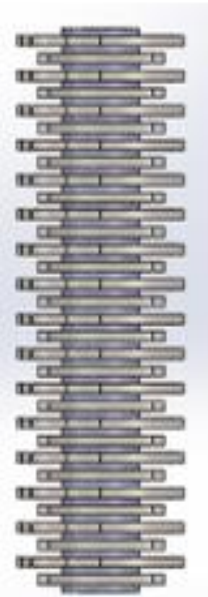

b)

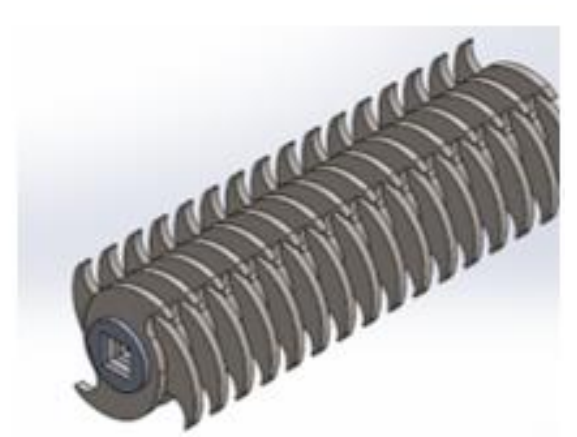

c)

La figura 3a, representa la vista frontal del juego de cuchillas base, tenemos una longitud de 148 $\mathrm{mm}$ y una altura de $50 \mathrm{~mm}$. El espesor de las cuchillas es de $6.4 \mathrm{~mm}$. Existe un separador entre cada cuchilla que tiene un espesor de $6.4 \mathrm{~mm}$. En la figura $3 \mathrm{~b}$, se muestra la vista superior donde van montadas las cuchillas base y los separadores, en total tenemos 33 cuchillas y 32 separadores, como consecuencia tiene una longitud de $366.40 \mathrm{~mm}$. Por último, la figura 3c, representa la vista isométrica del juego de cuchillas base. 
Figura 3. Juego de cuchillas base

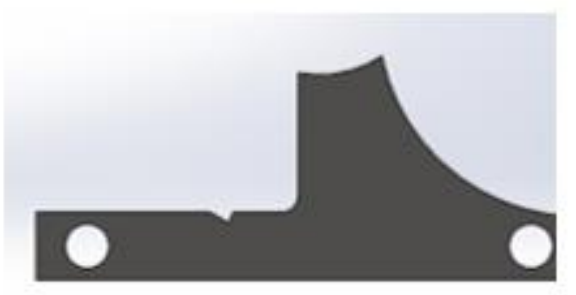

a)

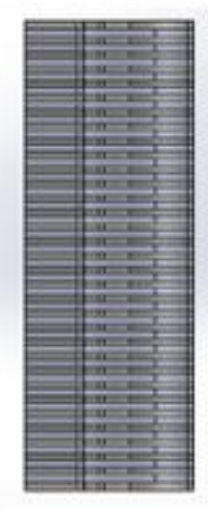

b)

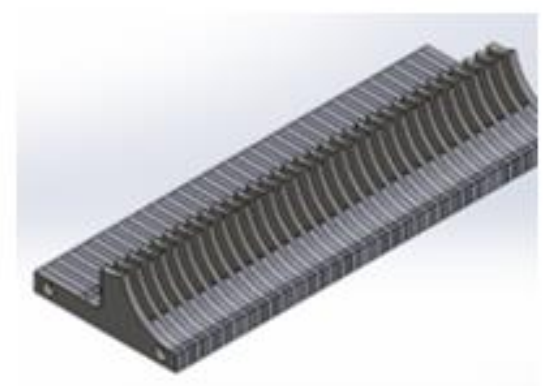

c)

La figura 4a, representa la vista frontal de la caja cortadora, tenemos una dimensión de largo de $196 \mathrm{~mm}$ por $132 \mathrm{~mm}$ de alto. En la figura 4b, se muestra la vista superior de la caja junto con el eje de transmisión, este tiene una longitud de $789 \mathrm{~mm}$. Por último, tenemos la figura 4c, que nos representa la vista isométrica de la caja cortadora y el eje de transmisión.

Figura 4. Armado de la caja cortadora

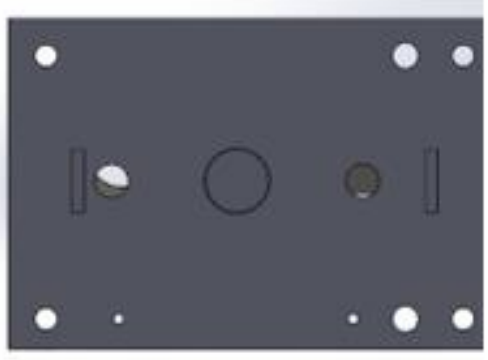

a)

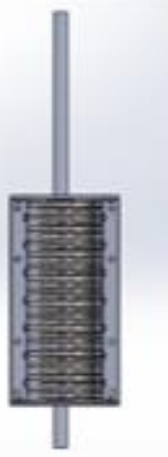

b)

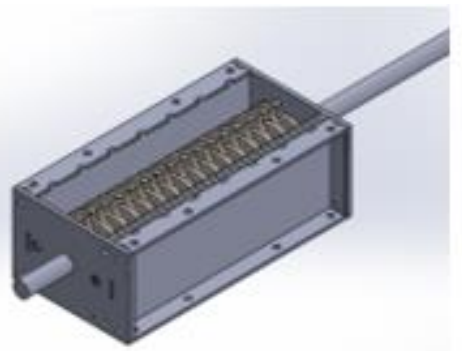

c)

En la figura 5, tenemos la base o mesa de soporte, dónde está montada la caja cortadora. La figura 5a, nos muestra la vista frontal, con dimensiones de largo $400 \mathrm{~mm}$ y de alto $1000 \mathrm{~mm}$. En la figura 5b, se muestra la vista lateral de la mesa de soporte, con dimensiones de $850 \mathrm{~mm}$ por $1000 \mathrm{~mm}$. Por último, tenemos la figura $5 \mathrm{c}$, que nos representa la vista isométrica de la mesa de soporte. 
Figura 5. Mesa de soporte

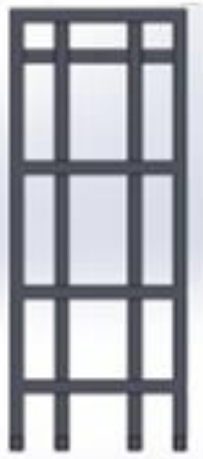

a)

b)

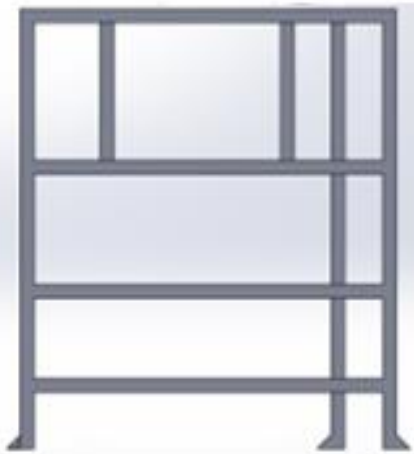

En la figura 6, tenemos las vistas principales representativas del molino compacto. La figura 6a, representa la vista posterior, donde se puede observar el conjunto de poleas, chumaceras, tolva de seguridad, mesa de soporte y las bandas de transmisión, para el funcionamiento de la máquina. En la figura $6 \mathrm{~b}$, se observa la vista superior y por último la figura $6 \mathrm{c}$, muestra la vista isométrica del molino compacto para triturar PET.

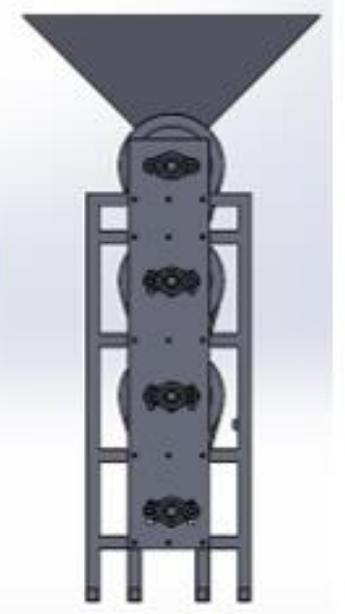

a)
Figura 6. Molino compacto para triturar PET

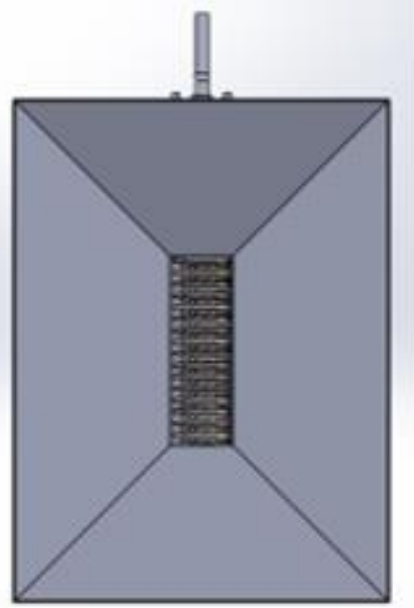

b)

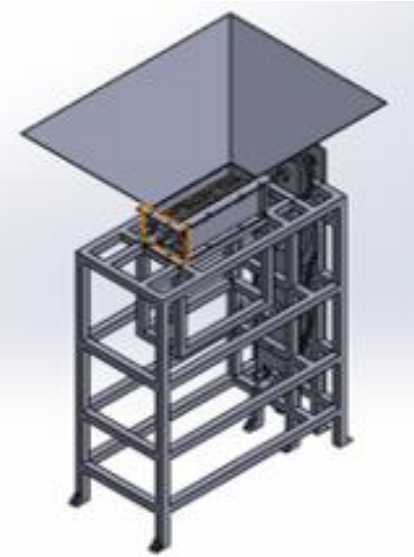

c)

\subsection{MATERIAL}

El material que se utilizará para construir las cuchillas principales del molino triturador de PET, es el acero inoxidable 316L, debido a que se realizó un estudio de análisis electroquímico de diferentes aceros inoxidables 416, 304 y 316L, siendo éste el que tuvo mejor comportamiento.

A continuación se muestra la comparación del comportamiento anódico de los aceros inoxidables 416,304 y $316 \mathrm{~L}$. 
Figura 7. Potencial V vs SCE.

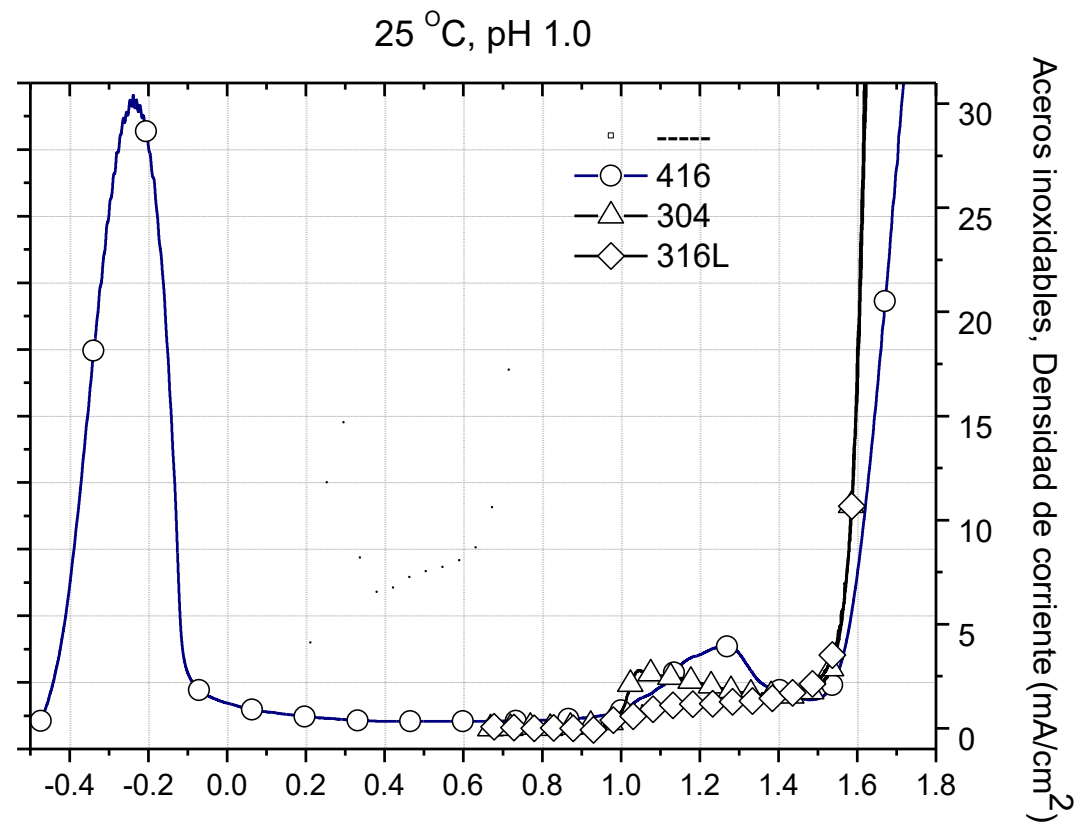

La figura 7, muestra las diferentes curvas del comportamiento anódico de tres aceros inoxidables, 416, 304 y 316L. Donde se observa el potencial aplicado en muestras de diferentes aceros sumergidos en una solución de $\mathrm{H} 2 \mathrm{O}-\mathrm{H} 2 \mathrm{SO} 4, \mathrm{pH} 1.0$, Temperatura ambiente $20^{\circ} \mathrm{C}$. También es posible observar la densidad de corriente y las zonas activo-pasivo-transpasivo de las tres curvas.

\subsection{GENERACIÓN DEL CÓDIGO “G”}

Se realizó el código G de la cuchilla principal por medio del software Aspire.

Figura 8. Generación del código G, en el software Aspire.

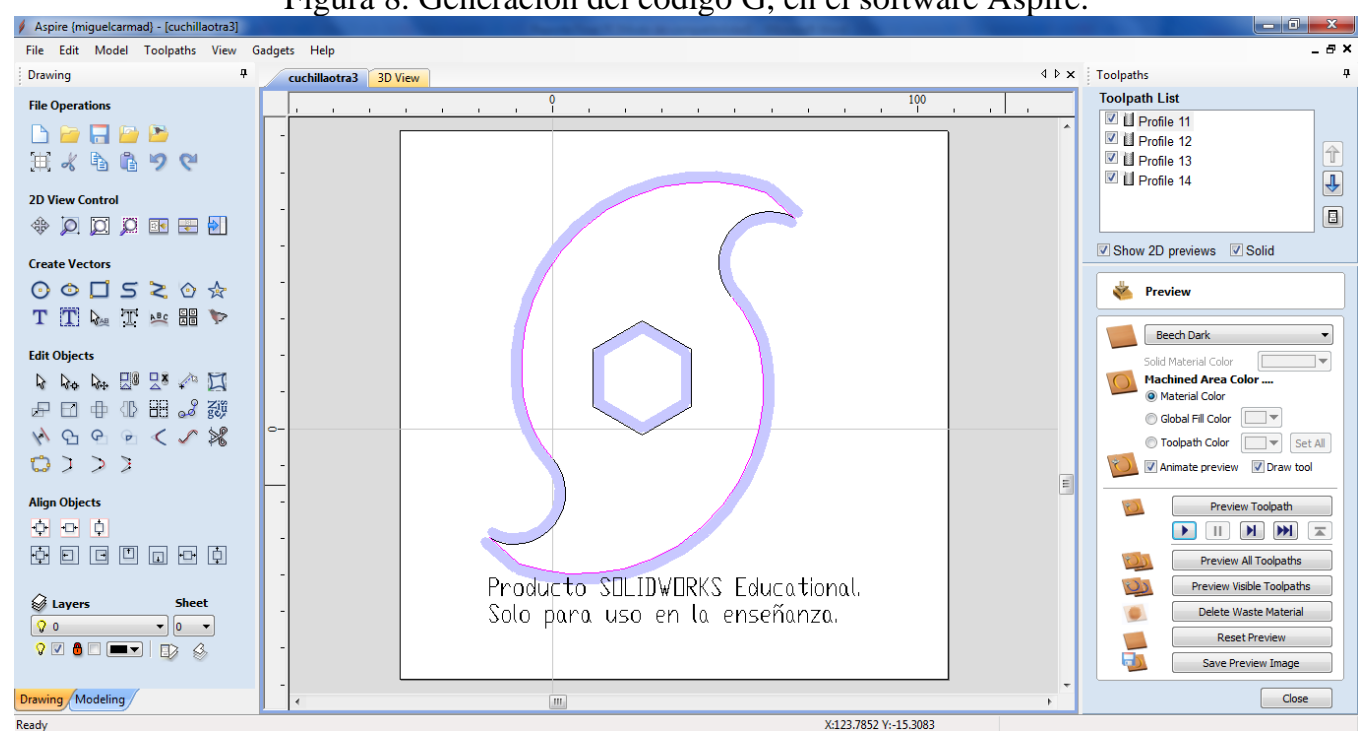


Este es el código G de la cuchilla principal.

Tabla 2. Código G de la cuchilla principal.

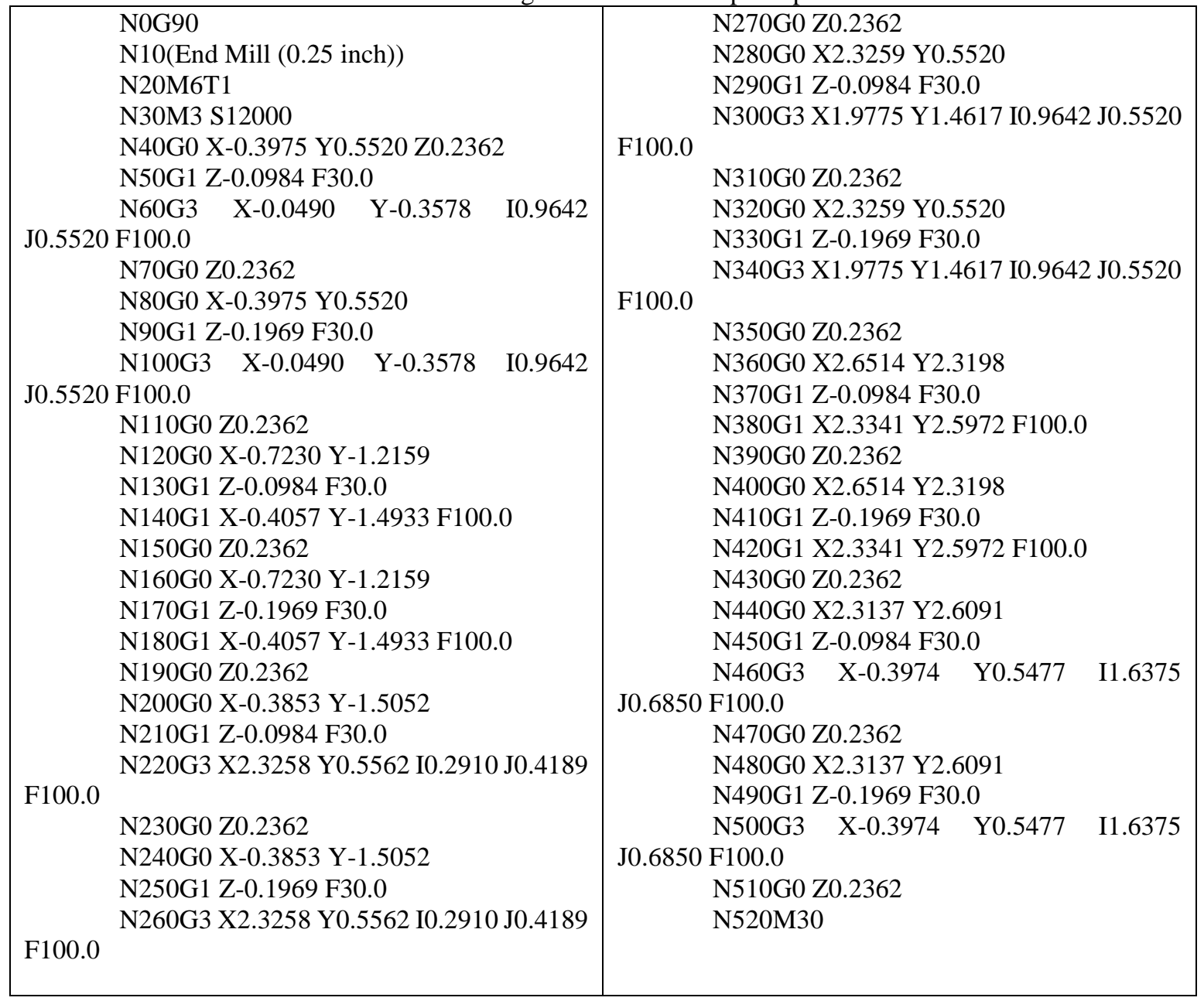

A continuación, se muestran los análisis que se realizaron por medio del elemento finito, mediante el software SolidWorks. En la figura 9. Se encuentra el análisis de Tensión de Von Mises. Se puede observar que las cuchillas no están en riesgo de fracturarse, debido a que el máximo esfuerzo que tienen es de 1.586E8 $\left(\mathrm{N} / \mathrm{m}^{2}\right)$ y el límite elástico del material de la cuchilla, que es de acero inoxidable 316L es de $1.7 \mathrm{E} 8\left(\mathrm{~N} / \mathrm{m}^{2}\right)$. Se puede decir que se tiene un factor de seguridad de $6.7 \%$, usando las cuchillas en su condición. 
Figura 9. Análisis de Tensión de Von Mises.

Nombre del modelo:cuchillaotra

Nombre de estudio:Análisis estático 1-Predeterminado-1

Tipo de resultado: Análisis estático tensión nodal Tensiones
Escala de deformación: 1
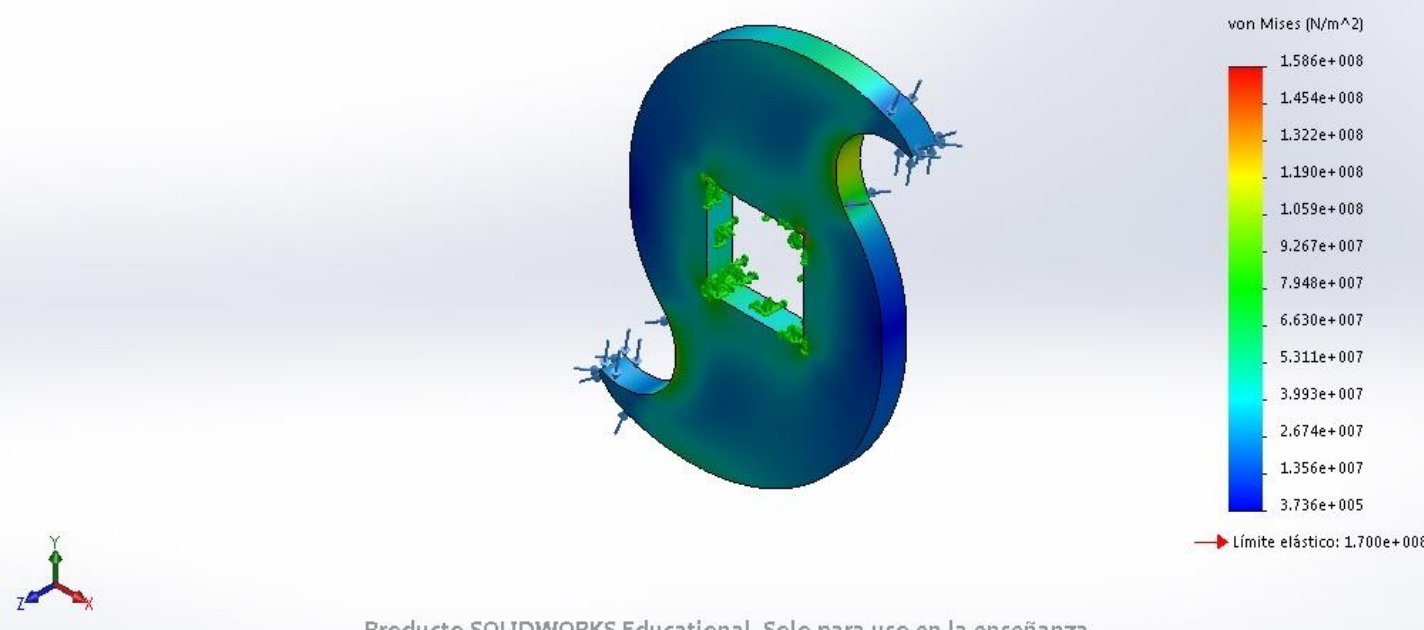

Producto SOLIDWORKS Educational. Solo para uso en la enseñanza.

En la figura 10. Se muestra el análisis de desplazamientos resultantes, se puede observar que el máximo desplazamiento resultante, se encuentra en la parte del filo de la cuchilla, que es de 0.0391547 $\mathrm{mm}$, pero este desplazamiento no es significativo para nuestro diseño, debido a que no alcanza ni un milímetro, por lo tanto no afectaría con las tolerancias establecidas en el diseño.

Figura 10. Análisis de desplazamientos resultantes.

Nombre del modelo:cuchillaotra3

1(-Predeterminado-

tico Desplazamientos1
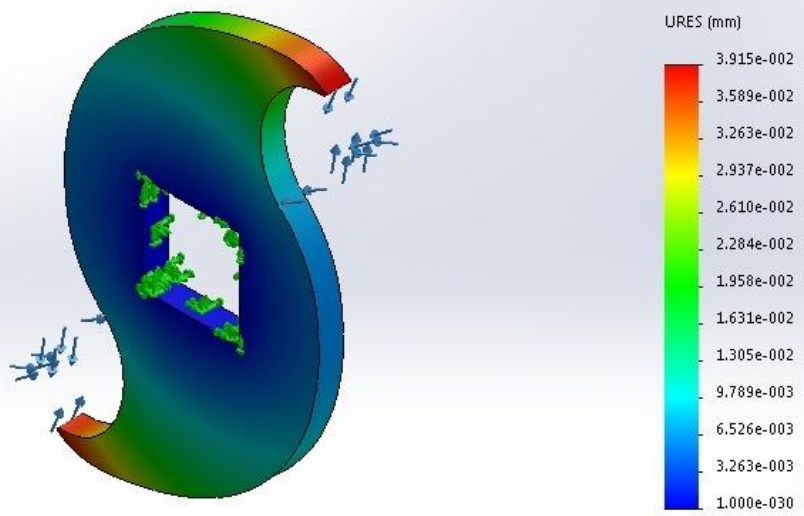

${ }_{2}$

Producto SOLIDWORKS Educational. Solo para uso en la enseñanza.

\section{CONCLUSIONES}

El uso de materiales poliméricos en la sociedad actual a crecido en forma exponencial, debido a la producción y uso. Sin embargo la utilización de polímeros, ha llevado a la sociedad a generar un gran 
problema de contaminación, particularmente el Polietileno de Teraftalato (PET). Para ello se vio la necesidad de diseñar y modelar un molino compacto con el programa SolidWorks.

Se desarrollaron todos los elementos de máquina del molino a través del programa SolidWorks, desde el inicio hasta el ensamble de cada uno de ellos. Se puede observar que no hay interferencia en cuanto a las partes que componen la máquina, lo que significa que, en la construcción en físico, no debería tener ningún problema en su funcionamiento.

Se obtuvo una gráfica del comportamiento anódico de diferentes aceros inoxidables, para la fabricación de las cuchillas, se determinó que, el acero 316L es el mejor material, debido a su resistencia electroquímica.

Se generó el código "G” para la fabricación de las cuchillas, en el software Aspire. Una vez que se realicen en físico, las cuales se van a realizar en una fresadora de control numérico. Los código se adaptaron para trabajar en una fresadora ExperMill VMC-0600.

Se realizó el análisis del elemento finito de las cuchillas en el software SolidWork, los análisis que se realizaron fueron el de Tensión de Von Mises y de Desplazamientos resultantes, en ninguno de los dos análisis existieron problemas, debido a que no se superó el límite elástico del material y también el desplazamiento resultante fue menos de medio milímetro.

En la actualidad podemos realizar el diseño y la simulación de una máquina, antes de realizarla en físico, esto nos ahorra bastante tiempo y dinero. Se puede asegurar que una vez fabricada, funcionará adecuadamente debido a que ya se realizó la simulación con los modelos o teorías adecuadas. 


\section{REFERENCIAS}

[1] Norton, R. L. (2011). Diseño de Máquinas. Un enfoque integrado. México: Pearson Educación.

[2] Rodríguez Vidal, C. (2015). Diseño mecánico con SolidWorks 2015: RA-MA Editorial.

[3] Wikipedia. (2021). Tereftalato de polietileno. Recuperado de https://es.wikipedia.org/wiki/Tereftalato_de_polietileno

[4] Norton, R. L. (1999). Diseño de Máquinas. México: Pearson Educación.

[5] Cornish Álvarez, M. (1997). El ABC de los plásticos. México: Pearson Educación. Universidad Iberoamericana.

[6] Cadena, F., \& Quiroz, F. (2000). Manual de reciclaje de plásticos. Quito Ecuador: Corporación Oikos.

[7] Hernández, R., \& Fernández, C. (2006). Metodología de la Investigación. México: McGraw-Hill Interamericana.

[8] Shigley, J.E. (1990). Diseño en Ingeniería Mecánica. México: McGraw-Hill.

[9] Mott, R.L. Diseño de Elementos de Máquinas. México: Prentice Hall.

[10] Askeland, D.R., \& Wright, W.J. (2000). Ciencia E Ingeniería de Los Materiales. México: CENGAGE Learning.

[11] Gómez, Sergio. (2020). El Gran Libro de Solidworks® Simulation. México: Alfaomega, Marcombo.

[12] Beer, F.P. (2009). Mecánica de Materiales. México: McGraw-Hill Educación. 